\title{
Some Basic Properties of Some Special Matrices. Part III $^{1}$
}

\author{
Xiquan Liang \\ Qingdao University of Science \\ and Technology \\ China
}

\author{
Tao Wang \\ Qingdao University of Science \\ and Technology \\ China
}

Summary. This article describes definitions of subsymmetric matrix, antisubsymmetric matrix, central symmetric matrix, symmetry circulant matrix and their basic properties.

MML identifier: MATRIX17, version: $\underline{7.12 .02 \quad 4.174 .1136}$

The notation and terminology used here have been introduced in the following papers: [7], [9], [13], [6], [14], [1], [3], [18], [17], [4], [2], [8], [11], [12], [16], [15], $[5]$, and [10].

\section{Basic Properties of Subordinate Symmetric Matrices}

For simplicity, we use the following convention: $n$ denotes a natural number, $K$ denotes a field, $a, b$ denote elements of $K, p, q$ denote finite sequences of elements of $K$, and $M_{1}, M_{2}$ denote square matrices over $K$ of dimension $n$.

Let $K$ be a field, let $n$ be a natural number, and let $M$ be a square matrix over $K$ of dimension $n$. We say that $M$ is subsymmetric if and only if:

(Def. 1) For all natural numbers $i, j, k, l$ such that $\langle i, j\rangle \in$ the indices of $M$ and $k=(n+1)-j$ and $l=(n+1)-i$ holds $M_{i, j}=M_{k, l}$.

Let us consider $n, K, a$. Note that $(a)^{n \times n}$ is subsymmetric.

Let us consider $n, K$. Observe that there exists a square matrix over $K$ of dimension $n$ which is subsymmetric.

\footnotetext{
${ }^{1}$ Authors thanks Andrzej Trybulec and Yatsuka Nakamura for the help during writing this article.

(C) 2012 University of Białystok CC-BY-SA License ver. 3.0 or later ISSN 1426-2630(p), 1898-9934(e)
} 
Let us consider $n, K$ and let $M$ be a subsymmetric square matrix over $K$ of dimension $n$. Note that $-M$ is subsymmetric.

Let us consider $n, K$ and let $M_{1}, M_{2}$ be subsymmetric square matrices over $K$ of dimension $n$. One can check that $M_{1}+M_{2}$ is subsymmetric.

Let us consider $n, K, a$ and let $M$ be a subsymmetric square matrix over $K$ of dimension $n$. Note that $a \cdot M$ is subsymmetric.

Let us consider $n, K$ and let $M_{1}, M_{2}$ be subsymmetric square matrices over $K$ of dimension $n$. One can verify that $M_{1}-M_{2}$ is subsymmetric.

Let us consider $n, K$ and let $M$ be a subsymmetric square matrix over $K$ of dimension $n$. Observe that $M^{\mathrm{T}}$ is subsymmetric.

Let us consider $n, K$. Observe that every square matrix over $K$ of dimension $n$ which is line circulant is also subsymmetric and every square matrix over $K$ of dimension $n$ which is column circulant is also subsymmetric.

Let $K$ be a field, let $n$ be a natural number, and let $M$ be a square matrix over $K$ of dimension $n$. We say that $M$ is anti-subsymmetric if and only if:

(Def. 2) For all natural numbers $i, j, k, l$ such that $\langle i, j\rangle \in$ the indices of $M$ and $k=(n+1)-j$ and $l=(n+1)-i$ holds $M_{i, j}=-M_{k, l}$.

Let us consider $n, K$. One can verify that there exists a square matrix over $K$ of dimension $n$ which is anti-subsymmetric.

The following proposition is true

(1) Let $K$ be a Fanoian field, $n, i, j, k, l$ be natural numbers, and $M_{1}$ be a square matrix over $K$ of dimension $n$. Suppose $\langle i, j\rangle \in$ the indices of $M_{1}$ and $i+j=n+1$ and $k=(n+1)-j$ and $l=(n+1)-i$ and $M_{1}$ is anti-subsymmetric. Then $\left(M_{1}\right)_{i, j}=0_{K}$.

Let us consider $n, K$ and let $M$ be an anti-subsymmetric square matrix over $K$ of dimension $n$. Note that $-M$ is anti-subsymmetric.

Let us consider $n, K$ and let $M_{1}, M_{2}$ be anti-subsymmetric square matrices over $K$ of dimension $n$. Observe that $M_{1}+M_{2}$ is anti-subsymmetric.

Let us consider $n, K, a$ and let $M$ be an anti-subsymmetric square matrix over $K$ of dimension $n$. One can verify that $a \cdot M$ is anti-subsymmetric.

Let us consider $n, K$ and let $M_{1}, M_{2}$ be anti-subsymmetric square matrices over $K$ of dimension $n$. One can check that $M_{1}-M_{2}$ is anti-subsymmetric.

Let us consider $n, K$ and let $M$ be an anti-subsymmetric square matrix over $K$ of dimension $n$. One can verify that $M^{\mathrm{T}}$ is anti-subsymmetric.

\section{Basic Properties of Central Symmetric Matrices}

Let $K$ be a field, let $n$ be a natural number, and let $M$ be a square matrix over $K$ of dimension $n$. We say that $M$ is central symmetric if and only if:

(Def. 3) For all natural numbers $i, j, k, l$ such that $\langle i, j\rangle \in$ the indices of $M$ and $k=(n+1)-i$ and $l=(n+1)-j$ holds $M_{i, j}=M_{k, l}$. 
Let us consider $n, K, a$. Note that $(a)^{n \times n}$ is central symmetric.

Let us consider $n, K$. One can verify that there exists a square matrix over $K$ of dimension $n$ which is central symmetric.

Let us consider $n, K$ and let $M$ be a central symmetric square matrix over $K$ of dimension $n$. One can verify that $-M$ is central symmetric.

Let us consider $n, K$ and let $M_{1}, M_{2}$ be central symmetric square matrices over $K$ of dimension $n$. One can verify that $M_{1}+M_{2}$ is central symmetric.

Let us consider $n, K, a$ and let $M$ be a central symmetric square matrix over $K$ of dimension $n$. Note that $a \cdot M$ is central symmetric.

Let us consider $n, K$ and let $M_{1}, M_{2}$ be central symmetric square matrices over $K$ of dimension $n$. Observe that $M_{1}-M_{2}$ is central symmetric.

Let us consider $n, K$ and let $M$ be a central symmetric square matrix over $K$ of dimension $n$. Observe that $M^{\mathrm{T}}$ is central symmetric.

Let us consider $n, K$. Note that every square matrix over $K$ of dimension $n$ which is symmetric and subsymmetric is also central symmetric.

\section{Basic Properties of Symmetric Circulant Matrices}

Let $K$ be a set, let $M$ be a matrix over $K$, and let $p$ be a finite sequence. We say that $M$ is symmetry circulant about $p$ if and only if the conditions (Def. 4 ) are satisfied.

(Def. 4)(i) $\quad \operatorname{len} p=\operatorname{width} M$,

(ii) for all natural numbers $i, j$ such that $\langle i, j\rangle \in$ the indices of $M$ and $i+j \neq \operatorname{len} p+1$ holds $M_{i, j}=p(((i+j)-1) \bmod \operatorname{len} p)$, and

(iii) for all natural numbers $i, j$ such that $\langle i, j\rangle \in$ the indices of $M$ and $i+j=\operatorname{len} p+1$ holds $M_{i, j}=p($ len $p)$.

The following propositions are true:

(2) $(a)^{n \times n}$ is symmetry circulant about $n \mapsto a$.

(3) If $M_{1}$ is symmetry circulant about $p$, then $a \cdot M_{1}$ is symmetry circulant about $a \cdot p$.

(4) If $M_{1}$ is symmetry circulant about $p$, then $-M_{1}$ is symmetry circulant about $-p$.

(5) If $M_{1}$ is symmetry circulant about $p$ and $M_{2}$ is symmetry circulant about $q$, then $M_{1}+M_{2}$ is symmetry circulant about $p+q$.

Let $K$ be a set and let $M$ be a matrix over $K$. We say that $M$ is symmetry circulant if and only if:

(Def. 5) There exists a finite sequence $p$ of elements of $K$ such that len $p=$ width $M$ and $M$ is symmetry circulant about $p$.

Let $K$ be a non empty set and let $p$ be a finite sequence of elements of $K$. We say that $p$ is first symmetry of circulant if and only if: 
(Def. 6) There exists a square matrix over $K$ of dimension len $p$ which is symmetry circulant about $p$.

Let $K$ be a non empty set and let $p$ be a finite sequence of elements of $K$. Let us assume that $p$ is first symmetry of circulant. The functor SCirc $p$ yielding a square matrix over $K$ of dimension len $p$ is defined as follows:

(Def. 7) SCirc $p$ is symmetry circulant about $p$.

Let us consider $n, K, a$. Note that $(a)^{n \times n}$ is symmetry circulant.

Let us consider $n, K$. Note that there exists a square matrix over $K$ of dimension $n$ which is symmetry circulant.

In the sequel $D$ is a non empty set, $t$ is a finite sequence of elements of $D$, and $A$ is a square matrix over $D$ of dimension $n$.

We now state the proposition

(6) Let $p$ be a finite sequence of elements of $D$. Suppose $0<n$ and $A$ is symmetry circulant about $p$. Then $A^{\mathrm{T}}$ is symmetry circulant about $p$.

Let us consider $n, K, a$ and let $M_{1}$ be a symmetry circulant square matrix over $K$ of dimension $n$. Note that $a \cdot M_{1}$ is symmetry circulant.

Let us consider $n, K$ and let $M_{1}, M_{2}$ be symmetry circulant square matrices over $K$ of dimension $n$. Note that $M_{1}+M_{2}$ is symmetry circulant.

Let us consider $n, K$ and let $M_{1}$ be a symmetry circulant square matrix over $K$ of dimension $n$. Note that $-M_{1}$ is symmetry circulant.

Let us consider $n, K$ and let $M_{1}, M_{2}$ be symmetry circulant square matrices over $K$ of dimension $n$. Observe that $M_{1}-M_{2}$ is symmetry circulant.

The following propositions are true:

(7) If $A$ is symmetry circulant and $n>0$, then $A^{\mathrm{T}}$ is symmetry circulant.

(8) If $p$ is first symmetry of circulant, then $-p$ is first symmetry of circulant.

(9) If $p$ is first symmetry of circulant, then $\operatorname{SCirc}(-p)=-\operatorname{SCirc} p$.

(10) Suppose $p$ is first symmetry of circulant and $q$ is first symmetry of circulant and len $p=\operatorname{len} q$. Then $p+q$ is first symmetry of circulant.

(11) If len $p=\operatorname{len} q$ and $p$ is first symmetry of circulant and $q$ is first symmetry of circulant, then $\operatorname{SCirc}(p+q)=\operatorname{SCirc} p+\operatorname{SCirc} q$.

(12) If $p$ is first symmetry of circulant, then $a \cdot p$ is first symmetry of circulant.

(13) If $p$ is first symmetry of circulant, then $\operatorname{SCirc}(a \cdot p)=a \cdot \operatorname{SCirc} p$.

(14) If $p$ is first symmetry of circulant, then $a \cdot \operatorname{SCirc} p+b \cdot \operatorname{SCirc} p=\operatorname{SCirc}((a+$ b) $\cdot p)$.

(15) If $p$ is first symmetry of circulant and $q$ is first symmetry of circulant and len $p=\operatorname{len} q$, then $a \cdot \operatorname{SCirc} p+a \cdot \operatorname{SCirc} q=\operatorname{SCirc}(a \cdot(p+q))$.

(16) Suppose $p$ is first symmetry of circulant and $q$ is first symmetry of circulant and len $p=\operatorname{len} q$. Then $a \cdot \operatorname{SCirc} p+b \cdot \operatorname{SCirc} q=\operatorname{SCirc}(a \cdot p+b \cdot q)$.

(17) If $M_{1}$ is symmetry circulant, then $M_{1}^{\mathrm{T}}=M_{1}$. 
Let us consider $n, K$. Note that every square matrix over $K$ of dimension $n$ which is symmetry circulant is also symmetric.

\section{REFERENCES}

[1] Grzegorz Bancerek and Krzysztof Hryniewiecki. Segments of natural numbers and finite sequences. Formalized Mathematics, 1(1):107-114, 1990.

[2] Czesław Byliński. Binary operations. Formalized Mathematics, 1(1):175-180, 1990.

[3] Czesław Byliński. Finite sequences and tuples of elements of a non-empty sets. Formalized Mathematics, 1(3):529-536, 1990.

[4] Czesław Byliński. Functions and their basic properties. Formalized Mathematics, 1(1):5565, 1990.

[5] Czesław Byliński. Partial functions. Formalized Mathematics, 1(2):357-367, 1990.

[6] Czesław Byliński. Some basic properties of sets. Formalized Mathematics, 1(1):47-53, 1990.

[7] Katarzyna Jankowska. Matrices. Abelian group of matrices. Formalized Mathematics, 2(4):475-480, 1991.

[8] Katarzyna Jankowska. Transpose matrices and groups of permutations. Formalized Mathematics, 2(5):711-717, 1991.

[9] Eugeniusz Kusak, Wojciech Leończuk, and Michał Muzalewski. Abelian groups, fields and vector spaces. Formalized Mathematics, 1(2):335-342, 1990.

[10] Karol Pąk. Basic properties of the rank of matrices over a field. Formalized Mathematics, 15(4):199-211, 2007, doi:10.2478/v10037-007-0024-5.

[11] Andrzej Trybulec. Binary operations applied to functions. Formalized Mathematics, 1(2):329-334, 1990.

[12] Michał J. Trybulec. Integers. Formalized Mathematics, 1(3):501-505, 1990.

[13] Wojciech A. Trybulec. Groups. Formalized Mathematics, 1(5):821-827, 1990.

[14] Zinaida Trybulec. Properties of subsets. Formalized Mathematics, 1(1):67-71, 1990.

[15] Edmund Woronowicz. Relations and their basic properties. Formalized Mathematics, 1(1):73-83, 1990.

[16] Xiaopeng Yue, Xiquan Liang, and Zhongpin Sun. Some properties of some special matrices. Formalized Mathematics, 13(4):541-547, 2005.

[17] Katarzyna Zawadzka. The sum and product of finite sequences of elements of a field. Formalized Mathematics, 3(2):205-211, 1992.

[18] Katarzyna Zawadzka. The product and the determinant of matrices with entries in a field. Formalized Mathematics, 4(1):1-8, 1993.

Received October 23, 2011 